\title{
L'ASTRONOMIE POPULAIRE, PRIORITÉ PHILOSOPHIQUE ET PROJET POLITIQUE
}

Dans une lettre à John Stuart Mill de 1844, Comte parle de «l'admirable impulsion philosophique que nos masses populaires ont indirectement reçue de notre grand ébranlement révolutionnaire ${ }^{1}$. L'expérience de plusieurs révolutions, qui place la France à l'avant-garde de la révolution occidentale, a fait des ouvriers parisiens un public particulièrement réceptif à la philosophie positive. Ainsi Comte justifie-t-il, auprès de J. Stuart Mill, l'intérêt soutenu des horlogers, mécaniciens, imprimeurs qui constituent, estime-t-il, environ un quart des auditeurs de son cours d'astronomie populaire.

Par ce jugement, Comte établit une connexion entre trois registres : la politique avec la question de l'héritage révolutionnaire, la philosophie positive avec le problème de sa diffusion et la science populaire avec l'évocation de son public. L'objet de cette étude sera de préciser ces relations par une lecture conjointe du Traité philosophique d'astronomie populaire, publié en 1844, et des études menées par les historiens sur les sciences pendant la Révolution. Il est hors de propos d'analyser dans son ensemble le contenu du Traité philosophique d'astronomie populaire, dont on ne lit généralement que le "Discours préliminaire" ou Discours sur l'esprit positif. Je me contenterai ici de soulever quelques problèmes relatifs à l'unité de ce Traité et à sa place dans l'œuvre comtienne. Dans une deuxième partie, je m'interrogerai sur le lien entre l'attitude de Comte et celle des révolutionnaires de l'An II. Enfin, en dégageant l'intention et la construction de ce Traité philosophique d'astronomie populaire, je m'efforcerai de montrer l'originalité de l'entreprise comtienne.

\footnotetext{
1. Auguste Cомте, Lettre à John Stuart Mill, du $1^{\text {er }}$ mai 1844 , Correspondance générale et confessions (cité par la suite comme Correspondance), Paris, Mouton/E.H.E.S.S., " Archives positivistes $\gg, 1975$, t. II, p. 248.
}

Revue de synthèse : IV S. Nº 1, janv.-mars 1991. 
Situé entre les deux œuvres monumentales de Comte, le Traité philosophique d'astronomie populaire, rédigé en 1844, apparaît comme une sorte d'interlude après la longue et pénible entreprise du Cours de philosophie positive (1830-1842) et avant les quatre tomes du Système de politique positive publiés de 1851 à 1854 . Le Traité n'est pas vraiment conçu mais seulement rédigé en 1844 , car il transcrit un cours oral d'astronomie populaire que Comte délivre depuis 1830 , le dimanche à la mairie du III $^{\mathrm{e}}$ arrondissement. Dans la Préface, Comte justifie ainsi l'ouvrage : « La publication de ces leçons m'ayant été souvent demandée, je me suis décidé à y consacrer l'une des rares intermittences de ma grande élaboration philosophique ${ }^{2}$.

Comte présente cet ouvrage didactique comme une parenthèse. De fait, le Traité ne trouve pas de place définie dans le plan de travail que Comte fixait en terminant le dernier tome de son Cours de philosophie positive, puisqu'il envisageait de mettre en marche la systématisation du positivisme par une série de quatre ouvrages : d'abord un de philosophie mathématique, et un ouvrage de politique, puis dans un deuxième temps un traité de l'éducation plus « un traité systématique de l'action de l'homme sur la nature ${ }^{3}$. De plus, ce Traité hors programme apparait comme un ouvrage mineur, un peu étouffé, éclipsé par le Discours sur l'esprit positif qui lui sert de préliminaire. On sait que Comte a retardé de quelques mois la publication du Traité, prévue pour mars 1844, et publié séparément trois cents exemplaires de son introduction, le «Discours préliminaire sur l'esprit positif ". Une lettre à J. Stuart Mill l'explique en ces termes:

« Je me suis décidé à publier séparément quelques exemplaires du discours préliminaire, qu'on imprime en ce moment, du petit Traité philosophique d'astronomie populaire, qui ne paraîtra qu'à la fin d'avril. Ce discours représente le discours d'ouverture de mon cours annuel [...]. En publiant à part ce discours, d'une centaine de pages, sous le titre de Discours sur l'esprit positif, je me suis proposé de donner une idée sommaire de la nouvelle philosophie à ceux qui ne veulent ou ne peuvent affronter la lecture des six énormes

2. ID., Traité philosophique d'astronomie populaire, 1844, Paris, Fayard, 1985, p. 9 ; cf. aussi Lettre du 23 déc. 1843 à J. Stuart Mill, in Correspondance, 1975, t. II, p. 224. Si l'on en juge d'après la correspondance, ce Traité serait presque une récréation après le «traité élémentaire de géométrie analytique » que Comte rédige aussi dans cette « intermittence " et qu'il trouve fort ennuyeux; cf. Lettre à $\mathbf{M}^{\text {me }}$ Comte, 12 déc. 1842 et J. Stuart Mill, 27 fév. 1843 , in Correspondance, 1975, t. II, p. 118, 126.

3. ID., Cours de philosophie positive (cité par la suite comme Cours), t. VI, 1842, Paris, Hermann, 1975, t. II, p. 790. 
volumes, dont toutes les principales conceptions y sont rapidement indiquées, avec un caractère convenable d'unité philosophique. C'est, en un mot, une sorte de manifeste systématique de la nouvelle école, et peut-être penserez-vous que, à ce titre, il comporte une véritable importance, indépendante de celle de l'ouvrage didactique dont il formera d'ailleurs le préambule ${ }^{4}$.

Certes, il est d'usage, depuis les Xvil et XviI ${ }^{e}$ siècles, qu'un traité scientifique soit précédé par un substantiel « discours préliminaire » exposant la philosophie de l'auteur, la situation encyclopédique de la science traitée et le plan de l'ouvrage. Mais les « Leçons d'astronomie " du Cours de philosophie positive, publiées en 1835 n'ont-elles pas déjà rempli cet office? Le discours préliminaire de Comte ne se conforme pas aux usages en vigueur dans ce genre littéraire. Il se donne comme « manifeste d'une école ", propagande d'un mouvement plus qu'introduction à l'astronomie. Le but est de toucher un large public, de mobiliser des forces, de faire des adeptes ${ }^{5}$. D'où la volonté de condenser en cent pages les six tomes du Cours ${ }^{6}$. Y a-t-il une réelle unité de l'ouvrage, plus largement, quels sont les liens entre le vaste projet philosophique de Comte et cet enseignement annuel d'astronomie, destiné aux masses?

La manière dont Comte parle de son Traité comme d'un ouvrage secondaire, accessoire, la manière dont il le publie, presque en appendice du Discours sur l'esprit positif, soulève quelques problèmes. Si Comte enseigne l'astronomie populaire, dix-sept ans durant, à titre bénévole, alors que son œuvre philosophique est déjà entravée par les diverses charges d'enseignement qu'il doit assurer pour sa subsistance, n'est-ce pas le signe que ce cours recouvre quelque intérêt ou enjeu considérables ? N'est-il pas significatif que lorsqu'il songe à rassembler ses disciples en une association, Comte la nomme d'abord « Association libre pour l'instruction positive du peuple dans tout l'Occident européen ", avant de l'appeler "Société positiviste ${ }^{7}$ ?

L'appel aux prolétaires est le motif qui fait de la science populaire un lieu privilégié dans la carrière de Comte. Au lieu de chercher à convertir à

4. A. Comte à J. Stuart Mill, le 6 fév. 1844, in Correspondance, 1975, t. II, p. 238.

5. Cf. ibid., p. 239 : A. Comte prie ici J. Stuart Mill « de placer utilement chez les cerveaux anglais qui vous paraîtront les mieux disposés » quelques-uns des trois cents exemplaires du Discours sur l'esprit positif édités séparément.

6. А. Сомте, op. cit. supra n. 2, p. 10.

7. ID., Lettres du 26 fév. 1848 et du 8 mars 1848, in Correspondance, 1981, t. IV, Annexes, p. 263-266 ; cf. Annie PeTr, " La diffusion des savoirs comme devoir positiviste ", Romantisme, $\mathrm{n}^{\circ}$ spécial « Sciences pour tous », 65, 1989, p. 7-25. 
la "saine philosophie " les hommes de science, comme il l'avait tenté, dès 1829 , dans son cours à l'Athénée, adressé à des professionnels de science, Comte se tourne exclusivement vers les prolétaires en même temps qu'il lance une campagne d'attaques contre les milieux scientifiques. Dès 1842, dans le dernier tome du Cours, Comte laissait entendre qu'il redoutait un phénomène de régression vers la scolastique et la métaphysique chez les mathématiciens contemporains : abus de l'esprit analytique, empirisme dispersif... sont, à ses yeux, des symptômes caractéristiques. Dans ses lettres de 1843, il parle à J. Stuart Mill d'une lutte interne à l'Académie des sciences entre les géomètres d'un côté et les biologistes de l'autre et il espère que les deux camps ennemis se tourneront vers la philosophie positive pour résoudre leur conflit ${ }^{8}$. Mais en 1844 , Comte ne croit plus à la possibilité d'une troisième force réunifiant le monde scientifique. Il désespère des savants professionnels, les condamne en bloc et critique vertement les institutions officielles : l'Académie des sciences, l'École polytechnique et les écoles de médecine? Comte leur reproche d'enfermer les études scientifiques dans d'étroites spécialités, de cultiver l'esprit de détail et de faire dégénérer l'esprit positif en s'opposant à toute généralisation ou synthèse des connaissances. Bref, un fossé est creusé entre deux termes jusqu'ici synonymes : « scientifique " et "positif». Les savants reconnus ne peuvent plus incarner l'esprit positif.

Il convient certes d'établir un rapprochement entre cette critique et les déboires de Comte à l'École polytechnique au début de 1844, en particulier ses mauvaises relations avec le puissant Arago, déjà professeur à Polytechnique au temps de Comte, secrétaire perpétuel de l'Académie des sciences depuis 1830 et rival direct de Comte dans l'exercice de l'astronomie populaire, puisque son cours à l'Observatoire attire les foules depuis $1812^{10}$.

Mais cet anti-académisme a des racines beaucoup plus profondes, qui remontent au temps de la Révolution. Comte ne manque pas de rappeler à ce propos, comme une mesure sage, quoique prématurée, le décret de fermeture des académies voté par la Convention nationale le 8 août 1793.

«L'instinct de progrès qui caractérisait, il y a un demi-siècle, le génie révolutionnaire, avait confusément senti ces dangers essentiels de manière à déterminer la suppression directe de ces compagnies arriérées, qui, ne convenant

8. A. Comme, Lettre à J. Stuart Mill du 27 fév. 1843, Correspondance, 1975, t. II, p. 140.

9. ID., Discours sur l'esprit positif, Paris, Fayard, 1985, p. 85.

10. Cf. Cours, préface personnelle au t. VI, $17-19$ juil. 1842 ; et Lettre au ministre de la Guerre, 25 janv. 1844, in Correspondance, 1975, t. II, p. 229-235; François ARAGo, Histoire de ma jeunesse, 1854, rééd. Paris, Christian Bourgois, 1985; et Maurice Daumas, Arago, la jeunesse de la science, Paris, Gallimard, 1943, rééd. Paris, Belin, 1987. 
qu'à l'élaboration préliminaire de l'esprit positif, devenaient de plus en plus hostiles à sa systématique finale. Quoique cette audacieuse mesure, si mal jugée d'ordinaire, fût alors prématurée, parce que ces graves inconvénients ne pouvaient encore être assez reconnus, il reste néanmoins certain que ces corporations scientifiques avaient déjà accompli le principal office que comportait leur nature : depuis leur restauration leur influence réelle a été, au fond, beaucoup plus nuisible qu'utile à la marche actuelle de la grande évolution mentale " ".

En 1848, lorsqu'il fonde la Société positiviste, Comte déclare qu'elle doit remplir « un office équivalent à celui qu'exerça si utilement la Société des Jacobins ${ }^{12}$. La réminiscence est explicite et mérite donc examen.

Dans quelle mesure le procès de la science académique et l'instauration d'un enseignement populaire font-ils de Comte un héritier des Jacobins qui triomphèrent en l'An II ? On sait que la fermeture des académies fut la conclusion d'une violente campagne contre les académies, orchestrée par Louis David et Marat qui harcèle les académiciens dans son journal L'Ami du peuple. Au milieu des injures et calomnies propres aux pamphlets de cette époque, on repère trois chefs principaux d'accusation contre l'Académie des sciences : 1) les académiciens sont des médiocres, des pédants privilégiés, des " charlatans modernes", dit Marat; 2) l'Académie est un cénacle fermé, plus préoccupé d'exclure et de condamner que de développer les sciences; 3 ) c'est une institution entièrement soumise à la monarchie qui reçoit les faveurs du roi et lui prête ses services ${ }^{13}$.

Les historiens s'accordent généralement à reconnaître que les motifs politiques ne suffisent pas à expliquer l'acharnement contre l'Académie des sciences. Marat, physicien et médecin, avait eu quelques déboires avec l'Académie en 1780 et sa théorie anti-newtonienne de la couleur fut alors ridiculisée par Laplace et Charles. L'explosion antiacadémique de l'An II, qui, par ailleurs, est contemporaine d'un grand essor des sciences naturelles et du Muséum, semble avoir quelque rapport avec un mouvement plus ou moins souterrain de contestation de la science newtonienne

11. A. Сомте, op. cit. supra n. 9, p. 85. Dès la $57^{2}$ leçon du Cours, à l'occasion d'une appréciation générale de l'œuvre de la Convention nationale, Comte formulait son opinion sur l'Académie des sciences en des termes semblables : " La suppression des académies, si amèrement déplorée par tant d'académiciens postérieurs, ne pouvait alors tenir essentiellement à de sauvages antipathies, mais bien plutôt à une certaine prévision générale, juste quoique confuse, des nouveaux besoins de l'esprit humain " (Cours, 1975, t. II, p. 594).

12. Cf. A. Comte, op. cit. supra n. 7, p. 265.

13. Cf. Roger HAHN, The Anatomy of a Scientific Institution: The Paris Academy of Science, 1666-1803, Berkeley, University of California Press, 1971, p. 227-285; voir aussi les réponses de Condorcet aux attaques contre l'Académie dans ses " Mémoires sur l'Instruction publique ", Euvres complètes, Paris, 1847-1849, t. VII, p. 295-308. Cf. Michel BLAY, " JeanPaul Marat, lecteur et traducteur de l'Opticks de Newton ", in Isaac Newron, Optique, Paris, Bourgeois, 1989, p. 433-454. 
dominante. On a souvent rappelé à l'occasion du Bicentenaire, l'interprétation suggérée dans les années 1950 par deux historiens américains : Henry Guerlac et Charles C. Gillispie ${ }^{14}$. Ils ont rapporté les mesures antiacadémiques des révolutionnaires à un conflit preexistant, déjà repérable dans l'Encyclopédie de Diderot, entre deux modèles de science : d'un côté, la science newtonienne, mathématisée, qui domine l'Académie et ne peut être cultivée que par un corps de savants hautement professionnalisés; de l'autre, les sciences naturelles, cultivées par Rousseau puis Bernardin de Saint-Pierre - directeur du Muséum en 1793 - sciences ouvertes à tous, professionnels ou amateurs. Bref, d'un côté la science aristocratique, de l'autre la science démocratique que, d'après Gillispie, les Jacobins auraient cherché à développer en créant des « Sociétés libres et fraternelles $" 15$.

Dans quelle mesure l'antiacadémisme virulent de Comte réactualise$t$-il ces conflits exacerbés à la fin du xvir ${ }^{e}$ siècle?

Il suffit de lire les pages de critiques virulentes dans la $57^{\mathrm{e}}$ leçon du Cours pour retrouver les accents de la campagne antiacadémique de l'An II. Comme Marat, Comte reproche aux académiciens leur « inqualifiable médiocrité ", leur " nature vulgaire " et il reprend même l'expression de "charlatanisme". Comte dénonce également un esprit de ligue chez les académiciens qui veillent à « se garantir les uns les autres, contre tout rival étranger, non seulement la possession d'honorables sinécures, juste équivalent des anciennes pensions, mais aussi et surtout le monopole universel du haut enseignement scientifique " ${ }^{16}$. En revanche, Comte ne reprend pas le reproche d'asservissement de l'Académie au pouvoir. Il tendrait plutôt à reprocher au gouvernement de laisser trop de pouvoir et d'autonomie aux savants. Aveuglés par leurs préjugés et leurs ambitions, ils sont incapables d'administrer correctement leurs propres institutions ${ }^{17}$.

Mais, par-delà cette divergence sur la liaison entre les savants et le pouvoir, Comte rejoint les révolutionnaires de l'An II par sa conviction de la portée politique de tout geste scientifique. Le choix d'un outil

14. Charles C. Gillispie, "The Encyclopédie and the Jacobin Philosophy of Science : A Study in Ideas and Consequences ", et la discussion qui suit cet exposé in Critical Problems in the History of Science, ed. Marshall Clagetr, Madison, University of Wisconsin Press, 1959 , p. 255-289.

15. La thèse de Gillispie, fortement marquée par le contexte de l'affaire Lyssenko dans les années 1950, a souvent été âprement critiquée car beaucoup de savants jacobins n'étaient pas antinewtoniens. Cf. Pietro REDONDI, « La Révolution française et l'histoire des sciences », $L a$ Recherche, 20, mars 1989, p. 320-331.

16. Cf. Cours, 1975, t. II, $57^{e}$ leçon, p. 631-632 sqq.

17. Cf., notamment, la critique de l'École polytechnique, in Cours, préface personnelle au t. VI, 1975, t. II, p. 470. 
mathématique, d'une méthode scientifique devient, pour Comte, un problème de société qui peut gravement retarder, voire menacer, le projet de réorganisation de l'Occident. Le statut des mathématiques semble nouer encore la parenté avec les Jacobins. Comte s'est toujours prononcé contre une mathématisation aveugle de toutes les sciences ${ }^{18}$; il déplore l'abus du discours algébrique et l'ésotérisme des langages scientifiques ${ }^{19}$; dans les années 1840 , il s'éloigne de plus en plus des mathématiciens et trouve dans les sciences de la vie des concepts et un modèle d'organisation plus proches de la positivité ${ }^{20}$.

Mais Comte ne cherche pas pour autant à réactiver la tradition naturaliste. Dans la $2^{e}$ leçon du Cours, il mettait à l'écart l'histoire naturelle, la géologie, la botanique, la zoologie, sciences dites concrètes, et, à ma connaissance, il n'est jamais revenu sur ce point dans la suite de ses œuvres. De plus, quand il critique les mathématiciens, Comte dénonce des abus mais ne remet pas en cause la nécessité des mathématiques dans les sciences de la nature et dans les études correspondantes. Quand il se fait fort d'enseigner l'astronomie à un public non instruit en mathématiques, il ne prétend pas qu'après avoir étudié ses 23 leçons, le lecteur sera au niveau d'un astronome de l'Observatoire. Loin de laisser entendre qu'on peut faire de l'astronomie sans mathématiques, en observant simplement le ciel, en amateur des étoiles, Comte dit que son objectif est de transmettre à tout lecteur, quel que soit son niveau d'études, le message minimum suivant : " chaque principale question astronomique est réductible à une recherche correspondante de géométrie ou de mécanique abstraites $"{ }^{21}$. Bien que, dans son analyse historique, Comte destitue peu à peu les mérites de Newton au bénéfice de Descartes, il n'a jamais contesté le modèle de la science newtonienne et laplacienne. Sa critique la plus sévère de l'École polytechnique, dans la Préface personnelle au tome VI du Cours, porte plus sur le fonctionnement administratif de l'institution - en particulier la composition du Conseil des professeurs qui mélange toutes les disciplines, sans distinction et sans égard pour la hiérarchie - que sur la science qu'on y enseigne ${ }^{22}$.

Enfin, troisième point, dans le procès de l'Académie et de l'École polytechnique, ne figure jamais l'accusation d'élitisme. Comte garde une conception assez aristocratique du savoir scientifique : il dénonce même violemment l'illusion des vulgarisateurs qui prétendent mettre la science

18. Cf. Cours, 1975, t. I, $28^{c}$ leçon, p. 448-452; ibid., $40^{c}$ leçon, p. $721-722$ et $44^{e}$ leçon, p. 832-833.

19. Ibid., $34^{e}$ leçon, p. 546.

20. Cf., en part., Cours, 1975, t. II, $58^{e}$ leçon, p. $701 s q$.

21. Cf. Traité philosophique d'astronomie populaire, op. cit. supra n. 2, p. 10.

22. Cf. Cours, préface personnelle au t. VI, 1975, t. II, p. 470, note. 
à la portée de tous et font croire qu'une intelligence médiocre peut s'élever jusqu' "aux plus hautes conceptions du génie humain " ${ }^{23}$. Il n'est donc pas question d'abolir la distance entre amateur et professionnel. S'il souhaite ouvrir le temple de la science au commun des mortels, Comte précise néanmoins que, faute d'instruction mathématique, certains devront se contenter d' "admettre de confiance " les résultats, de croire sur parole ${ }^{24}$. Partage du savoir, oui, mais la communion des esprits est impossible. L'instruction mathématique, la maîtrise des calculs infinitésimal et intégral, tracera toujours une ligne de clivage dans la société.

Et pourtant l'astronomie populaire de Comte est à bien des égards une entreprise révolutionnaire. J'entendrai ce mot, en deux sens. En un sens strictement politique, tout d'abord. Comte ne cache pas la finalité de son enseignement d'astronomie. Dès la " Préface », il parle de sa "destination à la fois mentale et sociale ".

«L'intention fondamentale qui doit aujourd'hui prévaloir dans une semblable élaboration didactique consiste à concevoir l'universelle initiation systématique de la raison publique à la saine philosophie astronomique, comme constituant un préambule indispensable, ou plutôt un premier degré normal, de l'établissement prochain d'un nouveau système philosophique pleinement homogène, seul susceptible désormais d'organiser des convictions durables et unanimes $"{ }^{25}$.

Propager la philosophie positive dans la classe populaire, préparer le consensus d'opinions qui fera la force du régime positif, c'est un programme à la fois philosophique et politique. Comte admet, en effet, qu'un régime positif ne peut s'instaurer que par une "régénération ", une sorte de rééducation des savants académiciens jugés déviants. Il organise pour cela un " appel direct et soutenu au bon sens universel » qui est la source de toute science positive. Grâce à l'enseignement populaire, les prolétaires qui, pour Comte, incarnent le bon sens, se réapproprient en quelque sorte le capital culturel qui a fructifié dans les équations et corporations savantes.

Ce contact entre les savants et le peuple doit exercer une heureuse réaction en retour sur le progrès scientifique en le normalisant et le régulant. Une foule de "controverses oiseuses ou dangereuses, que nous a

23. Cf. Cours, $24^{e}$ leçon (1830), 1975, t. I, p. 380-381.

24. Cf. Traité philosophique d'astronomie populaire, op. cit. supra n. 2, p. 10.

25. Ibid., p. 12. 
laissées l'Ancien Régime mental ", devraient ainsi disparaître. Comte attend de la science populaire qu'elle crée un garde-fou contre les excès de spécialisation. Le prolétaire instruit d'astronomie sera le censeur des savants enclins à l'ésotérisme ou à la dispersion, le gardien de l'esprit positif. Le public de la science populaire doit même s'ériger en « un vaste tribunal spontané, aussi impartial qu'irrécusable $"{ }^{26}$.

Peut-on imaginer proposition plus subversive? Non seulement les savants réunis en académies n'ont plus le monopole de l'autorité scientifique mais au lieu d'exercer eux-mêmes la fonction de juges au nom de la science ils seront à leur tour jugés par un tribunal populaire. C'est une proposition inouïe, qui dépasse en hardiesse celles des Jacobins insurgés contre les académies. En effet, Comte place les savants sous contrôle et transforme les académies en quartiers de haute surveillance. En ce sens, l'astronomie populaire esquisse une révolution culturelle, en profondeur et sur le long terme.

Mais la route est longue pour atteindre ce régime de savoir. Il n'est pas question de livrer aux prolétaires, appelés à contrôler les productions savantes, une science au rabais, un vernis de culture scientifique. Encore moins de les émerveiller en présentant le spectacle du ciel étoilé. Il faut une longue éducation, une lente maturation des esprits qui, reproduisant l'itinéraire accompli par l'humanité, élève l'esprit des travailleurs jusqu'aux sommets de la science. Le cours d'astronomie populaire n'est qu'un premier pas dans l'initiation encyclopédique et il coûte déjà vingttrois semaines d'efforts.

L'entreprise de régénération est un long processus qui exige toute une suite de préparations. Ceci est déjà sensible au niveau du Discours sur l'esprit positif, dans l'exposé de la célèbre loi de hiérarchie des sciences ${ }^{27}$. $\mathrm{Au}$ lieu de présenter un ordre linéaire de préséance, Comte suggère une classification en système. L'astronomie n'est plus présentée comme une science autonome mais comme élément dans un ensemble, unique corpus de savoir, la science de l'humanité. L'astronomie est la base d'une pyramide dont la pointe est la sociologie. Elle devient une sorte de propédeutique.

Dans le Traité lui-même, la pédagogie comtienne est sans complaisance et tout en exigences. Au lieu de suivre une progression didactique linéaire, Comte multiplie les détours et médiations. Sans entrer ici dans l'étude détaillée de la construction du Traité qui a été faite ailleurs, je rappellerai seulement ce soin obsédant des préliminaires ${ }^{28}$. Aux « apprécia-

26. Cf. Discours sur l'esprit positif, op. cit. supra n. 9, p. 54, 86.

27. Ibid., p. 107.

28. Cf. Bernadette Bensaude-VINCENT, "Auguste Comte, la science populaire d'un philosophe ", Corpus, revue de philosophie, 4, 1987, p. 143-167. 
tions préliminaires" succèdent les "préambules indispensables", " premières ébauches ", " sommaires indications ", " revues préalables". Comme si chaque chapitre ne faisait que préparer le terrain; jusqu'au dernier qui recule encore le moment d'atteindre à l'essentiel en renvoyant le lecteur aux ouvrages spécialisés. Tout le Traité philosophique d'astronomie populaire est ainsi construit comme une sorte de spirale : chaque circonvolution encercle ou circonscrit la science astronomique. L'ensemble des chapitres constitue un long travail d'approche, comme s'il fallait pour apprivoiser le ciel, mimer le mouvement des astres.

Voici, après la première figure jacobine de la révolution, une deuxième idée de révolution immergée dans le cours populaire de Comte : non plus rupture radicale qui subvertit l'ordre établi mais mouvement circulaire, réglé par des lois, et défini par ses conditions initiales. Le temps de l'éducation populaire est celui d'une lente initiation qui parcourt toutes les révolutions du savoir, qui prépare les esprits, et mûrit le sens commun. C'est pour Comte le temps qu'il faut pour une véritable révolution. Jamais les explosions violentes, les émeutes et barricades ne pourront instaurer dans la société un changement véritable, un changement durable. Le ressort de l'efficacité se trouve dans les vertus des longs processus démultipliant les préliminaires. En ce XIX siècle agité de secousses révolutionnaires, qui célèbre la vitesse et l'accélération du progrès, Comte invite à la lenteur.

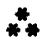

Le Traité philosophique d'astronomie populaire est donc une œuvre capitale parce qu'il constitue un tournant, à double titre.

Tournant dans la pensée de Comte. Situé à la charnière entre ses "deux carrières ", il montre comment s'opère le déplacement de l'élaboration philosophique vers les problèmes d'organisation politique. Avec la science populaire émerge une nouvelle problématique, celle de la diffusion de la philosophie positive et de son public; un nouveau style d'analyse, en termes de rapport de forces dans la société ; et un nouveau souci : faire des adeptes, mobiliser les masses.

Le Traité marque aussi un tournant dans la gestion de l'héritage révolutionnaire. Par sa critique des académies, par son souci de la popularisation, Comte retrouve certains accents des révolutionnaires de l'An II. Sa contestation de la science officielle diffère cependant : Comte ne rêve pas d'une alternative à la science professionnelle, il ne conteste pas la légiti- 
mité du discours des experts. Mais il leur refuse le monopole du savoir. Faire de la science une chose publique, c'est préparer la véritable révolution.

Bernadette Bensaude-VINCENT

Université Paris- $X$. 